\title{
Activités
}

5-1 | avril 2008

Varia

Contribution d'une analyse du discours à l'étude des stratégies d'actions et de régulation des agents en situation d'écoute téléphonique

L'exemple du dispositif d'urgence sociale 115

Contribution of speech analysis to the study of action and regulation strategies

of agents working in crisis phone service: the case of the French homeless hotline

115

Contribución de un análisis del discurso al estudio de las estrategias de acción y de regulación de los agentes en situación de escucha telefónica : el ejemplo del dispositivo de urgencia social 115

\section{Sounkalo Djibo}

\section{(2penEdition}

Journals

Édition électronique

URL : http://journals.openedition.org/activites/1898

DOI : 10.4000/activites. 1898

ISSN : 1765-2723

Éditeur

ARPACT - Association Recherches et Pratiques sur les ACTivités

\section{Référence électronique}

Sounkalo Djibo, «Contribution d'une analyse du discours à l'étude des stratégies d'actions et de régulation des agents en situation d'écoute téléphonique », Activités [En ligne], 5-1 | avril 2008, mis en ligne le 15 avril 2008, consulté le 30 avril 2019. URL : http://journals.openedition.org/activites/1898; DOI : 10.4000/activites. 1898

\section{(c) (i) $\ominus$}

Activités est mis à disposition selon les termes de la licence Creative Commons Attribution - Pas d'Utilisation Commerciale - Pas de Modification 4.0 International. 


\title{
Contribution d'une analyse du discours à l'étude des stratégies d'actions et de régulation des agents en situation d'écoute téléphonique : l'exemple du dispositif d'urgence sociale 115
}

\author{
Sounkalo Djibo \\ Laboratoire ECCHAT-Contacts \\ l’Université de Picardie Jules Verne 80025 Amiens Cedex. \\ sounkalo.djibo@u-picardie.fr
}

\begin{abstract}
Contribution of speech analysis to the study of action and regulation strategies of agents working in crisis phone service: the case of the French homeless hotline 115. In this study we analyzed the representations of listener-operators of the emergency hotline " 115 " for the homeless, to assess the difficulties linked to their work and the strategies which they implement to deal with them. Our analysis was based on recorded interviews relating listener-operator experiences. The relation involved in assistance by telephone requires a number of skills in a range of fields. However it appears to be based on a series of paradoxes which impact considerably on the listening activity. Other factors of an organizational nature also add to the work's difficulties, such as polluting calls having no direct connection with the assistance activity, hotline dependence on other social structures, etc. In this situation, the 115 hotline agents develop individual and collective strategies to better manage constraints resulting from certain calls or lack of places in the shelter program.
\end{abstract}

KEYWORDS

Emergency hotline ; assistance by telephone ; speech analysis ; individual and collective strategies.

\section{1.- Introduction}

Les activités téléphoniques occupent une place importante parmi les travaux consacrés au travail à distance, dont la particularité est d'éloigner les agents de la situation classique de travail et d'impliquer des adaptations nouvelles (Trognon, 2002). Le mérite de ces travaux c'est d'avoir montré l'importance que recouvrent les activités téléphoniques dans l'émergence de nouvelles formes de métiers et les exigences qu'elles supposent en termes de formation et d'adaptation. Ainsi, l'activité téléphonique peut être perçue comme une activité de gestion des flux d'appels, autrement dit une activité cognitive et sociale de coproduction de connaissance circonstancielle, mobilisant un savoir technique et des compétences communicatives (Falzon, 1994; Falzon, \& Lapeyrière, 1998; Brangier, 2002; Minondo, 2002). Elle peut également concerner l'assistance en mettant en perspective des activités dans lesquelles un expert propose une aide à un consultant. Cette deuxième approche recouvre plusieurs situations professionnelles que sont l'assistance informatique (Lambolez, 2002) le conseil administratif (Brangier, 2002), le soutien aux personnes malades (Engrand, 2002; Minondo, 2002) ou en grande détresse (Lancry-Hoestland, 2002). L'aide aux personnes en détresse par le téléphone rassemble selon Cerf et Falzon (2005), « un ensemble d'activités destinées à aider des personnes en difficulté socio-économique à construire leur capacité d'action en respectant un système de lois, de règles et de normes d'une société donnée. L'activité s'exerce directement auprès des individus dans des lieux (parfois) mal circonscrits (la rue) et consiste à faire valoir auprès de ces individus leurs 
droits mais aussi leurs devoirs afin d'engendrer un changement dans leurs comportements...Cette activité nécessite des compétences de diagnostic et de conduite d'une relation à caractère éducatif. Le développement de ces compétences est en grande partie expérientiel et nécessite que les agents apprennent à partager leurs expériences ».

Avec la loi de décentralisation (1982), l'activité sociale a connu un développement considérable, évalué par un certain nombre d'études orientées pour la plupart, vers l'état des lieux de ce secteur ou bien encore la qualification des emplois des personnes œuvrant dans le social (Favart-Drialud, 1991 ; Davidson, Francescon, Negroni, Pezet-Langevin, \& Rolland, 1995; Chopart, 2000; Chauvière, 2002). Ces études abordent pour la plupart des problématiques de dialogues entre les agents et les personnes qui appellent. Marchand et Navarro (1994) ont analysé le contenu des dialogues entre permanenciers du SAMU et les appelants, mettant en évidence l'usage d'un vocabulaire différent dans les dialogues en fonction du type d'appelants (particuliers ou spécialistes). Les spécialistes ont ainsi un discours plus structuré que celui des particuliers. Pour sa part, Engrand (2002) a analysé des extraits de discours issus d'un dialogue téléphonique dans le cadre d'une relation d'aide au téléphone sur le cancer. L'auteur montre que le traitement de la demande implique l'explication de la démarche de l'appelant et la reconnaissance progressive du discours de celui-ci. Autrement dit, la gestion de l'activité est co-construite à distance (Trognon, \& Kostulski, 2000). Dans le même ordre d'idées, Minondo (2002) a cherché à expliquer la variabilité du contrôle de la compréhension et de son positionnement dans le dialogue en fonction de la complexité de la situation. L'analyse qui a porté sur des permanenciers du Service d'Aide Médicale d'Urgence (SAMU) a mis en évidence l'importance des caractéristiques de l'interlocuteur au plan de ses compétences communicatives et de la complexité de la tâche pour la mise en œuvre du contrôle de la compréhension du permanencier.

Dans une tout autre direction d'autres études ont porté sur les aspects affectifs de la relation d'écoute téléphonique. C'est le cas notamment de la contribution de De Gaulmyn $(1994 ; 2002)$ au sein de laquelle, l'expression et les reformulations d'émotions manifestées et perçues dans l'écoute, dans le cadre d'un service d'urgence sociale, ont été analysées. Ces études montrent que les écoutants cacheraient leurs émotions par peur de s'humilier lorsqu'ils sont en interaction avec les personnes qui appellent.

Malgré l'intérêt qu'elle suscite, l'activité d'aide par le téléphone et par extension le secteur social a mobilisé très peu d'études ergonomiques (Villatte, Teiger, \& Caroly-Flageul, 2004). Les contributions significatives ont le plus souvent porté sur le thème de l'usure professionnelle des travailleurs sociaux (Pezet-Langevin, 1997, 2001; Trichot, 2004). Les obstacles à une telle entreprise tiennent d'une part, à la spécificité des demandes sociales souvent éloignées des préoccupations de l'ergonomie, et d'autre part aux caractéristiques du champ qui rendent difficiles les interventions. En effet, ces demandes portent pour la plupart sur la professionnalisation, notamment la reconnaissance des compétences, le statut, mais aussi la formation, le déroulement des carrières, etc. (Lancry-Hoestland, 2002). De l'autre côté, les obstacles sont d'ordre organisationnel: prescription floue du travail, diversité des pratiques, difficulté pour les travailleurs à expliquer leur travail, secteur très chargé d'émotions d'où autocensure, et tensions pouvant conduire à l'usure professionnelle (Dejours, 1993; Gbézo, 2000; Trichot, 2004).

La présente étude a été réalisée en banlieue parisienne. À partir de la demande d'un dispositif d'urgence sociale confronté souvent à un turn-over important dans son service d'écoute téléphonique, nous avons été mandatés pour proposer des recommandations utiles non seulement pour caractériser l'activité des agents, mais aussi pallier les difficultés d'ordre organisationnel auxquelles ils étaient confrontés. Le présent article se propose de restituer une partie de cette étude, notamment l'analyse de l'activité réalisée essentiellement à partir du discours des agents dont le rôle consiste à proposer au téléphone un hébergement d'urgence à des personnes sans abris. Ce choix méthodologique a été fait faute de pouvoir observer le travail en continu. Dans cette perspective, nous avons cherché à comprendre le vécu de ces agents, notamment le rapport aux difficultés pendant l'exécution de leur 
tâche ${ }^{1}$. Nous nous sommes attachés principalement à évaluer les difficultés de la relation d'écoute téléphonique et les stratégies déployées par les écoutants pour réguler leur activité, selon qu'il s'agisse de femmes ou d'hommes en charge de l'écoute. Cette distinction nous a semblé importante dans la mesure où elle permettait de cerner les différences de modes opératoires mis en œuvre par les agents venant compenser ainsi la rareté des études ergonomiques entreprises dans cette direction (Messing, 1999). La problématique de cette étude interroge donc la relation d'écoute téléphonique dans ses conditions de réalisation. Elle consiste à montrer que cette relation dépend de plusieurs facteurs liés notamment à l'expertise des agents, mais aussi à l'organisation du travail.

\section{2.- La relation d'aide au téléphone}

La relation d'aide s'est considérablement développée ces dernières années pour s'étendre à plusieurs secteurs du social en France: enfance en danger, femmes victimes de violence conjugale ou bien encore la question du logement social, etc. Conçus au départ comme de simples relais d'information, ces différents services se sont très vite développés pour devenir incontournables face à la détresse sociale. Leur point commun c'est d'être des situations isolées de travail mettant en relation téléphonique des opérateurs et des populations difficiles (Chauvière, 2001; 2002; Lechat, \& Delaunay, 2003). Pour la plupart, il a été mis en place des numéros de téléphone abrégés, ce qui facilite leur accès par les usagers. Malgré leur utilité publique et leur popularité, ces services sont aussi souvent en proie à des difficultés liées non seulement aux problématiques des populations qui les sollicitent, mais aussi à des aspects organisationnels. Cela s'exprime notamment par une charge de travail et une charge émotionnelle importantes, car les écoutants gèrent des appels dont certains polluent les lignes téléphoniques et les exposent parfois à des actes d'incivilité lorsque la demande n'est pas satisfaite (injures, humiliations, etc.). Ce qui n'est pas sans conséquences sur leur santé psychique.

La téléphonie sociale est née du monde de la solidarité dans les années 60, à l'initiative des associations militantes puis des services de l'Etat (Cadéac, Ferranini, \& Oddoux, 2004). Avec le temps, elle est devenue incontournable au point d'intégrer les dispositifs de prévention et d'accompagner les interventions traditionnelles du social, du psychologique et du médical. Mais au-delà de ce rôle de proximité, la téléphonie sociale accompagne également les décisions de l'Etat en matière de politique de santé publique. Malgré le développement que la téléphonie sociale a connu, il existe très peu d'études qui lui ont été consacrées. Les plus significatives sont celles de Bange et De Gaulmyn (1990) sur l'analyse qualitative des interactions téléphoniques et la récente publication de l'Institut National de la Prévention et d'Education pour la Santé (INPES). Les raisons sont essentiellement liées à l'importance et à la diversité des services dédiés, à la confidentialité qui entoure l'exploitation de la documentation, et à l'anonymat voulu par les écoutants sur le contenu de leur écoute (Delignières, 2002).

\section{1.- Une diversité de services pour un objectif commun}

À la suite de l'enquête de l'INPES, on dénombre plus de 24 services téléphoniques nationaux d'information, d'orientation et de soutien dédiés aux addictions, aux maladies chroniques, à la sexualité, à la contraception, à la santé des jeunes, aux droits des personnes, à la santé mentale, etc. Chacun de ces services est investi de missions et pratiques spécifiques. Bien qu'organisés en pôles thématiques (sida, suicide, tabac, cancer, drogues, etc.) ou populationnels (enfance maltraitée, femmes victimes de violences, personnes âgées, etc.), ils ont pour objectif principal d'aider les personnes qui appellent à faire face à la situation, en leur apportant l'information ou bien le soutien recherché.

Si la téléphonie sociale est basée sur l'écoute, elle s'appuie en réalité sur des fondements théoriques en interaction les uns avec les autres: relation d'aide, communication, psychologie cognitivo-com-

1. Cette étude a fait l'objet d'une communication au Congrès international « Homme et organisation: la santé au cœur des enjeux des entreprises » tenu du 22 au 24 novembre 2005 à Nancy, Metz (France) et à Aix-les-Bains (Luxem-

bourg) : www.travailetsante.org 
portementaliste, psychanalyse, etc. De sorte qu'il est très rare que l'activité s'appuie exclusivement sur une seule de ces théories. Dans la pratique, l'écoute téléphonique correspond à une fonction clinique spécifique dans le sens où, les procédures usuelles que suppose la relation de face à face disparaissent. Il s'agit avant tout d'une recherche de lien, d'une présence d'autrui (Montheil, 1997). Ainsi, le nom de la ligne d'écoute suffit à lui seul pour énoncer le problème à traiter. Par ailleurs, c'est l'appelant qui structure le dialogue, même si de temps en temps, l'écoutant peut intervenir ponctuellement. En effet, l'appelant peut décider de stopper l'appel ou rappeler une autre fois, etc. Dans cette relation où la voix constitue le seul support, les associations d'idées sont favorisées. Car, comme l'écrit Demoulin (1996), «à partir de la mise à distance des corps de l'appelant et de l'écoutant, grâce à la protection de l'anonymat, quelque chose d'indicible ailleurs peut tenter de se formuler».

Les informations sur les statistiques des appels en matière de téléphonie sociale sont difficilement accessibles. Il s'agit le plus souvent de chiffres utilisés en interne par les services, ce qui leur permet d'ajuster leur activité. Le moins qu'on puisse dire, c'est que le nombre d'appels vers les services dédiés a sensiblement augmenté dans l'ensemble. Par exemple, «Ligne de vie », un service d'écoute et d'accompagnement d'aide en matière d'éducation thérapeutique sur le SIDA, le cancer et l'hépatite indique dans son rapport d'activités, une progression de 2150 à 3102 appels, soit plus de $44 \%$ d'appels entre 1997, date de sa création, et l'année 2000. Selon ce même rapport, les hommes représentent $58 \%$ des appelants, et parmi ceux-ci, c'est la classe d'âge de 30-39 ans qui semble la plus concernée. Parmi les thèmes conversationnels abordés, les difficultés psychologiques et thérapeutiques sont les plus courantes. Il faut aussi mentionner les catégories des appels, dont une grande majorité n'aboutit pas. Cela est dû aux flux des appels à des moments clefs de la journée. S'ils aboutissent, certains des appels polluent les lignes téléphoniques. C'est le cas notamment des appels muets, des blagues, des menaces, des insultes, etc. Ils représentent une proportion considérable pouvant aller jusqu'à $40 \%$ des appels selon le service téléphonique.

L'opacité qui entoure les statistiques des appels téléphoniques prévaut également au sujet de l'origine socioprofessionnelle des écoutants et de leurs compétences. On dénombre toutefois deux catégories: des bénévoles et des professionnels de l'écoute. Les écoutants bénévoles sont les premiers à avoir apporté leur aide aux personnes en détresse au téléphone. Le plus souvent, ils ont connu plusieurs services et acquis une solide expérience dans le domaine de l'écoute. Par exemple, la charte de Sida Info Service, expose clairement cette intention: «Les écoutants répondants » ne sont pas des délégués d'une profession dans un service téléphonique mais ils mettent au service de l'écoute leurs qualités acquises par ailleurs: l'écoute est à elle seule une qualification riche de toutes les qualifications individuelles ». La deuxième catégorie est apparue à partir des années 80 . Selon l'enquête de Lehnisch (1986), les trois quarts des écoutants appartiennent au monde du travail social et parmi eux un tiers sont des psychologues.

\section{2.- Le service d'hébergement d'urgence par le téléphone}

Le service d'aide au logement par téléphone communément appelé Samu Social a été créé en 1995 sous le numéro vert 0800306 306. Deux ans plus tard, il a cédé sa place au numéro d'urgence gratuit « 115 ». Depuis cette date, ce service a connu des records d'affluence au point de ne plus pouvoir s'adapter à la demande d'hébergement. Mais à s'intéresser de plus près, on constate que les maux dont souffre ce dispositif relèvent en grande partie de problèmes organisationnels.

\subsection{1.- Une gestion décentralisée}

Le service d'urgence social est accessible partout en France en composant le même numéro (115). Par contre, sa gestion est « départementalisée », tant dans son financement que dans l'organisation interne du travail des écoutants. Le financement des activités du 115 relève de l'Etat, plus précisément de la Direction Départementale des Affaires Sanitaires et Sociales (DDASS) qui oriente les objectifs. Quant à la gestion effective, elle est confiée le plus souvent à des Associations loi 1901 travaillant 
dans le sens de la réinsertion sociale. En effet, sur les neuf dispositifs d'urgence sociale que comptent Paris et sa banlieue, trois gestionnaires du 115 sur quatre sont des associations. Chaque dispositif est autonome et organise à sa façon l'activité de l'écoute. Autrement dit, il y a n'y a pas d'harmonisation des pratiques entre les différents gestionnaires. Par exemple, l'organisation des horaires d'écoute diffère d'un département à un autre. Pendant que certains dispositifs fonctionnent jour et nuit, d'autres par contre privilégient une période, par exemple la journée, pour répondre aux appels. Le nombre d'écoutants par plage horaire n'est pas le même non plus d'un dispositif à un autre. Par ailleurs, l'équipement en outils informatiques et les logiciels utilisés pour évaluer et gérer les appels sont assez disparates. Si certains de ces logiciels sont simples d'utilisation d'autres par contre sont contraignants, parce que nécessitant beaucoup d'informations à chaque évaluation. Autant d'éléments qui ne permettent pas d'objectiver les différentes statistiques adressées périodiquement à la DDASS.

Pourtant, un Observatoire national du 115 a été créé depuis 1997 par l'Etat et confié à la Fédération Nationale des Associations de Réinsertion Sociale (FNARS), avec pour objectifs de recueillir, analyser et diffuser les données disponibles sur le 115 (Les Info du 115, 2003). Lors de la journée nationale consacrée aux enjeux de l'observation sociale en décembre 2003, cette orientation a été de nouveau réaffirmée. C'est ainsi qu'on apprend que le recueil des données sur le 115 a une logique transversale. L'analyse des données doit permettre de « fédérer, coordonner, mettre en réseau ».

C'est à cette occasion également qu'une véritable topographie des appels a été faite. Sur la base de deux séries d'observations menées en novembre 2002 et avril 2003, une étude sur les " appels véritables ${ }^{2}$ » a été réalisée sur 55 départements, à l'exception de Paris. Les résultats montrent un taux d'appels variant d'un département à un autre et confirment la précédente évaluation réalisée en 1999. Cette étude révèle que $71 \%$ des appels véritables sont composés par les usagers, et les autres par des tiers, notamment les travailleurs sociaux (15\%). Les appels véritables sont le plus souvent composés en début de semaine, entre 16 heures et 20 heures, et très peu en week-end. Ils sont composés en majorité par des hommes seuls (63\%), âgés de 25 à 60 ans. On observe toutefois une proportion non négligeable de femmes seules (16\%). Le reste des appels concerne des familles notamment des couples avec des enfants (4\%), des couples sans enfant (5\%) et des femmes seules avec des enfants (7\%). Si l'hébergement constitue la principale source des appels, les demandes d'informations et de droits regroupent une part non négligeable (12\%).

\subsection{2.- Les difficultés liées à ce service}

Au-delà de ces aspects purement institutionnels, un certain nombre de difficultés impactent le travail des agents de l'hébergement d'urgence. Ces difficultés sont liées à la conjugaison de plusieurs facteurs, notamment la saturation des lignes téléphoniques, et l'inadaptation des lieux d'accueil.

La saturation des lignes concerne la téléphonie sociale en général. Toutefois, ce problème est encore plus accru concernant l'hébergement d'urgence, en raison des demandes d'hébergement de plus en plus nombreuses. Ce phénomène s'observe le plus souvent en période hivernale, du fait de la surmédiatisation du dispositif par les pouvoirs publics. Une des limites du dispositif que reconnait la FNARS dans son rapport d'activité 2003-2004, qui évalue à $48 \%$ seulement, le taux des « appels décrochés ${ }^{3}$. Un chiffre dérisoire surtout lorsque l'on considère que l'Institut National des Statistiques et des Études Économiques (INSEE) estimait à 63500 adultes et 16000 enfants le nombre de personnes sans domicile en 2002. C'est majoritairement une population issue des classes populaires qui ont perdu leur logement à la suite d'une séparation d'avec le conjoint, de changement de région ou de pays (29\% d'étrangers), de l'impossibilité de régler le loyer ou victime d'une expulsion du logement. Plus d'un quart des personnes sans abris (des jeunes de moins de 30 ans) n'a jamais eu de

2. Les appels véritables concernent les appels porteurs d'une demande pouvant venir des usagers ou bien de tiers (travailleurs sociaux, ou particuliers) et qui aboutissent à une écoute, contrairement aux appels polluants (silence, insultes ou simulation de personnes sans abri).

3. Les « appels décrochés » concernent les appels qui aboutissent et qui ont subit un traitement par les écoutants, y compris les appels polluants (simulation de sans abri, insultes, etc.). 
logement, a connu une enfance difficile: famille éclatée, placement en famille d'accueil ou en foyer. Si la majorité est au chômage (43\%), 29\% travaille et $28 \%$ est inactive.

Une réalité qui contraste avec les possibilités d'hébergement. Car, malgré la volonté affichée de l'État, l'ensemble du dispositif d'hébergement d'urgence de la région parisienne est saturé. Lors de la remise du rapport annuel du Haut Comité pour le logement des personnes défavorisées, Xavier Emmanuelli, le président fondateur du Samu Social affirmait que «le dispositif d'hébergement est aujourd'hui en crise » (Chambras, 2004). Ainsi, les centres d'hébergements, couplés aux hôtels sociaux et privés, n'arrivent plus à satisfaire les nombreuses demandes, même pendant le renfort hivernal du dispositif d'urgence. Certaines catégories de demandes ne trouvent pas d'offre adaptée (exemple des usagers fragilisés sur le plan sanitaire). L'une des raisons de cette saturation est à mettre au compte de la difficulté d'accès à un logement ordinaire. Le plus souvent, lorsque les demandes aboutissent, les hébergés se voient obligés de faire appel encore au 115 le lendemain, pour espérer trouver un toit pour la nuit suivante. Il s'ensuit donc un ballottage de structure d'hébergement en structure d'hébergement, tout au long de l'année, jusqu'à ce qu'une admission dans un foyer social, pour quelques mois ne leur soit proposée. Une situation qui fragilise davantage les personnes sans abri, surtout lorsque l'on considère que près de $30 \%$ d'entre eux souffrent de problèmes psychologiques (Pliez, 2003). Si l'on fait abstraction de l'inadaptation de certains centres d'hébergement, la plupart dépasse le nombre d'admissions autorisées. L'incendie de l'hôtel social à Paris qui a causé la mort de 24 demandeurs d'asile, le vendredi 15 avril 2005, démontre cet anachronisme. En effet, plus de 90 personnes étaient logées dans une structure dont la capacité d'accueil est de 60 personnes (Caillat, 2005).

\section{3.- Méthodologie}

Avant d'exposer la méthodologie de cette étude, nous définissons la tâche des écoutants et procédons à une présentation du dispositif d'urgence où nous avons effectué nos observations.

\section{1.- Tâche des écoutants}

La tâche des écoutants 115 consiste à écouter, informer et orienter les personnes sans abris. Cette tâche s'organise autour d'un certain nombre d'opérations:

Identification de la demande: cela consiste à voir s'il s'agit d'un habitué du dispositif ou pas. Ce diagnostic se fait progressivement par le jeu des questions-réponses. Lorsqu'il s'agit d'un habitué, le diagnostic est très simple, l'activité se résumant à obtenir des informations supplémentaires telles que les démarches auprès du travailleur social qui suit l'intéressé. Dans le cas contraire, une évaluation de la demande est faite selon les critères du logiciel utilisé par les écoutants : identité, situation familiale (seule ou en famille), zone d'appel, situation professionnelle, motif de l'appel (problème de ressources, expulsion, etc.), suivi social ou pas, temps d'errance, etc.

Recherche de l'information souhaitée par interrogation du dispositif: cette opération consiste à rechercher une place qui correspond non seulement aux souhaits de l'usager mais surtout à sa problématique. En effet, il s'agit d'une population hétéroclite, allant des personnes en grande errance, à des femmes victimes de violence, en passant par des demandeurs d'asile et des personnes expulsées de leur logement. L'objectif pour les écoutants c'est de trouver la solution la mieux indiquée, de manière à ce que l'usager soit entouré de l'ensemble des prestations sociales et / ou psychologiques nécessaires à sa réinsertion, une fois accueilli dans un centre d'hébergement d'urgence. Pour cela, les écoutants consultent les disponibilités au moment de l'appel. S'il n'existe aucune place, ils vont appeler les différents centres d'hébergement pour leur exposer la situation. Cette démarche peut se solder par un échec malheureusement.

Transmission de la réponse à l'usager: lorsque le dispositif est en mesure d'accueillir l'usager, les écoutants informent en priorité la structure susceptible de l'accueil, en transmettant toutefois les 
informations récoltées lors de l'évaluation. L'importance d'une telle démarche c'est de permettre dans un premier temps à la structure, d'en être informée, ce qui évite de questionner plusieurs fois l'intéressé une fois sur place. Un rendez-vous est pris pour que l'accueil se fasse dans les normes et en présence d'un animateur sur les lieux.

Un dialogue s'instaure entre l'écoutant et l'usager. Sa durée peut dépendre de plusieurs facteurs, par exemple la nature et le contenu de cette demande, le fait qu'il s'agisse d'une personne qui recherche un hébergement pour la première fois ou non. La réponse peut être positive ou négative dans le cas où le dispositif n'aurait plus de place. Parfois, lorsqu'elle est négative, certains usagers peuvent développer des réactions agressives.

\section{2.- Les aspects organisationnels du dispositif d'urgence}

\subsection{1.- Les conditions de travail}

Le dispositif d'urgence où cette étude a été menée est composé d'écoutants permanents travaillant la plupart du temps à mi-temps, étant donné l'organisation du travail qui les conduit à être sur les lieux parfois en journée, parfois la nuit voire le week-end. Une situation atypique du travail qui les oblige à s'adapter à des horaires alternants. En plus de ces écoutants, une autre catégorie de personnel qualifiée quant à elle de conjoncturelle, vient en appui en cas de défaillance des écoutants permanents pour cause de maladie ou de congés.

L'activité des écoutants 115 est basée à plus de 90\% sur des outils de communication:

Un standard téléphonique aux multiples fonctions. Il permet en effet une prise de casque, une écoute amplifiée, une mise en attente musicale de l'appelant lorsque l'écoutant est déjà en conversation avec un autre appelant, le transfert des appels polluants vers une messagerie vocale afin de dissuader les plaisantins de tels agissements, etc.;

Un autre téléphone, leur permet de joindre les collègues de l'équipe mobile SAMU le soir venu, ou bien de recevoir occasionnellement des appels des différentes structures d'hébergement pour des disponibilités. Ce téléphone permet aussi de contacter les collègues écoutants d'autres départements pour obtenir plus d'informations sur un usager, compte tenu de la grande mobilité des personnes sans abri;

Un fax permet l'envoi des taux d'occupation des structures d'hébergement à la DDASS, la réservation de places dans des hôtels conventionnés et la réception des disponibilités des différents centres d'hébergement;

Une photocopieuse leur permet régulièrement de faire des copies des différents documents de prises de notes, notamment les fiches de contact sur lesquelles ils évaluent la demande des personnes sans abris;

Enfin, un ordinateur type PC leur sert d'outil d'évaluation des appels.

Cette activité s'étend aussi à des tâches de rédaction de rapports, de cahier de consignes, d'établissement de statistiques, etc. Ainsi, comme l'ont évoqué Villate, Teiger et Caroly-Flageul (2004) à propos du travail du social en général, l'activité des écoutants 115 va au-delà de la simple relation d'écoute, pour reposer sur certaines composantes administratives.

Le dispositif que nous avons interrogé a une capacité d'hébergement limitée en comparaison avec la demande de plus en plus importante. Il est constamment sollicité, avec près de 1000 appels jour d'origine très variée: 


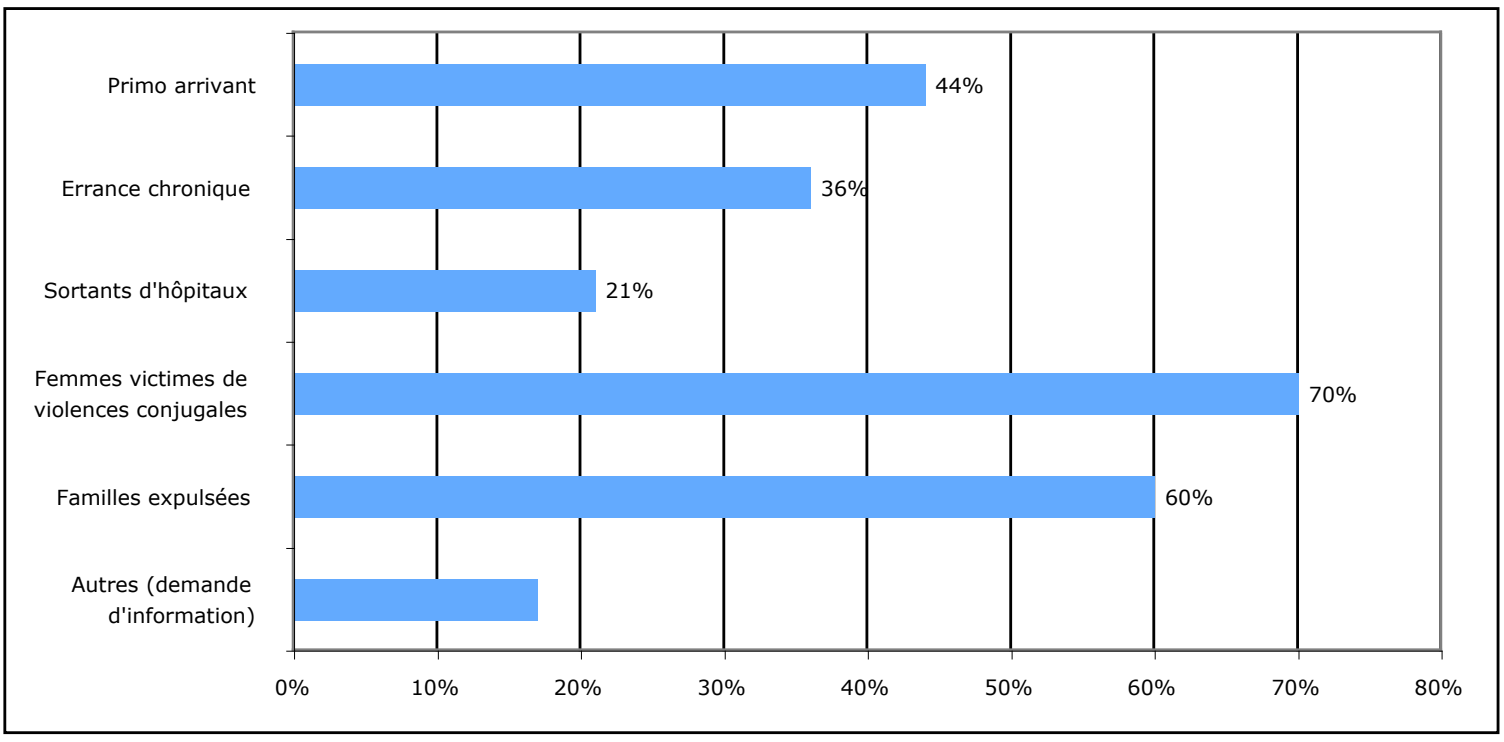

Figure 1: Statistiques des appels sur le dispositif d'urgence

Figure 1: Statistics relating to calls made via the emergency hotline

Comme on le perçoit sur ce graphique, les appels des personnes en errance chronique constituent la part importante des appels, soit 36\%. En seconde position, on trouve les appels concernant les personnes en fin de séjour à l'hôpital, soit $21 \%$. Viennent ensuite les appels autres que la problématique de l'hébergement, par exemple la demande d'information, soit 17\%. Les appels initiés par des personnes primo arrivant de l'étranger s'élèvent eux aussi à hauteur de $14 \%$. Cette catégorie concerne le plus souvent des personnes demandeuses d'asile. Les femmes victimes de violence conjugale regroupent quant à elles $7 \%$ des appels sur ce dispositif. Enfin, on enregistre un pourcentage de familles expulsées de leur logement de l'ordre de 5\% également.

Pour faire face à cette charge de travail, les écoutants sont répartis sur trois types de plages horaires:

La plage de $8 \mathrm{~h}$ à $14 \mathrm{~h} 30$ au cours de laquelle ils évaluent les demandes de personnes seules ou en présence d'un travailleur social. L'échange dans une telle situation est riche car elle permet de mieux cerner les contours du problème et les différentes démarches entreprises par la personne demandeuse. En outre les zones d'ombres, notamment celles qui ne peuvent être cernées durant une évaluation en l'absence d'un travailleur social, peuvent être levées.

Celle de $14 \mathrm{~h} 30$ à $21 \mathrm{~h}$ est de même nature que la précédente. La référence aux travailleurs sociaux est en effet appréciée. Ce qui permet de mieux cerner la problématique du demandeur. Elle permet l'écoute et l'orientation des personnes le plus souvent vers des places d'urgence ou à l'hôtel pour une semaine.

La plage horaire de $20 \mathrm{~h} 30$ à $8 \mathrm{~h} 30 \mathrm{~h}$ est la plus longue et la plus incertaine du point de vue de l'interface avec les personnes sans abris. Cette période est quelques fois source d'angoisse de part et d'autre de la relation entre les écoutants et les personnes qui font les demandes d'hébergement. Du côté des personnes sans abris, cette angoisse est liée à la possibilité d'obtenir une réponse négative. Chez les écoutants cette angoisse liée non seulement au fait de travailler seul durant cette période sans aucune possibilité de recours, mais aussi au manque de places. En début de soirée, on observe une affluence des appels, notamment ceux qui dans la journée n'ont pas eu satisfaction et ont été invités à renouveler leurs appels en cours de soirée. Une fois que les places ont été toutes distribuées, l'activité des écoutants est réduite à de la simple écoute. Cela se passe en fin de soirée et concerne le plus souvent des personnes en proie à une grande souffrance existentielle qui veulent tout simplement être écoutées. 
Les conditions de travail exposées ci-dessus influent également sur la qualité de l'écoute. Ce que nous démontrons dans les lignes qui suivent.

\subsection{2.- Les conditions de l'écoute}

Le travail des écoutants apparaît ainsi comme une gestion rigoureuse d'informations. Il faut rechercher ces informations à travers l'évaluation de la demande et l'interrogation du dispositif. Comme l'a démontré Boucheix (1991) dans un tout autre domaine, il s'agit donc d'une construction de la demande en vue de constituer un référentiel commun à l'usager et à l'écoutant. Suivant cette logique, les écoutants de l'hébergement d'urgence agissent sur un dispositif somme toute indépendant de leurs actions.

En outre, leur activité dépend de facteurs comme les plages horaires, denses au cours de la journée et souples au cours de la soirée, même si elles peuvent être source d'angoisse dans ce dernier cas. Les conditions climatiques déterminent également l'activité des écoutants. L'hiver est la période la plus redoutée, car propice aux appels. Avec l'arrivée du printemps, beaucoup de centres d'hébergement sont fermés paradoxalement au moment où les expulsions locatives pour impayés se multiplient. Quelles que soient les conditions de travail, le traitement d'un appel en durée est variable et peut avoisiner 5 à $10 \mathrm{mn}$. Il peut aller quelquefois au-delà, lorsqu'il s'agit d'une nouvelle évaluation, ou bien lorsqu'elle a lieu en relation avec un travailleur social.

L'évaluation est faite sans contact direct avec la personne. Il faut savoir poser les bonnes questions sans heurter la sensibilité des usagers. Il importe également d'identifier le plus rapidement possible une personne qui a déjà appelé, afin d'éviter de lui poser les mêmes questions. À défaut, cela est mal vécu par les personnes sans abri. L'activité au 115 suppose un coût cognitif important à gérer. Car la navigation entre les vrais appels et les appels polluants est source de stress pour eux, ce d'autant plus que pour l'instant, le dispositif ne dispose d'aucun moyen pour identifier les vrais appels des faux. De plus, les écoutants doivent être constamment disponibles et ne doivent pas céder à l'énervement face ces appels polluants.

\section{3.- Le recueil des données}

Les données ont été récoltées à partir de la méthode de l'entretien. À défaut de pouvoir observer en continu l'activité des écoutants, nous avons fait le choix des entretiens qui, de notre avis, étaient les plus indiqués pour exprimer leur vécu. La durée moyenne d'un entretien était d'une demi-heure environ. Enregistré sur une cassette audio avec l'accord des opérateurs, chaque entretien comportait trois volets:

Le premier volet concernait la présentation de l'écoutant. Il répondait notamment aux questions ouvertes sur son parcours professionnel et son statut au sein du dispositif;

Le second volet concernait l'organisation du travail. L'opérateur répondait aux questions portant sur les conditions de travail, les horaires, etc.;

Le troisième volet de l'entretien a porté sur les difficultés rencontrées au cours de l'écoute. Enfin, l'écoutant était questionné sur les aménagements qu'il pensait indispensables à faire pour pallier les difficultés liées à l'écoute.

Au final, 32 entretiens ont été réalisés dont 18 avec des hommes et 14 avec des femmes.

\section{4.- L'analyse des données}

Dans un premier temps, nous avons retranscrit l'ensemble des entretiens en respectant autant que possible la chronologie des événements.

Dans un second temps, le matériel d'analyse a été encodé à l'aide du logiciel Alceste (Reinert, 1987). Alceste est un logiciel de traitement de données textuelles permettant de quantifier un texte pour 
en extraire les structures signifiantes les plus fortes, afin de dégager l'information essentielle. Ces structures signifiantes sont regroupées sous la forme de classes de discours. Le matériel a été encodé pour chaque opérateur suivant les trois volets de l'entretien décrits précédemment (présentation; organisation; difficultés de l'écoute téléphonique). Les variables ci-après ont été également prises en compte lors de l'encodage:

Le sexe de l'opérateur, pour voir l'impact d'une telle activité en fonction du genre des écoutants;

L'expertise, pour mesurer l'impact des connaissances et des savoir-faire des écoutants sur la gestion des émotions;

Le service occupé (jour; nuit; week-end), pour identifier les moments propices à l'apparition des polluants;

L'antécédent de l'opérateur (travailleur social; étudiant; autre) pour voir s'il existe une représentation différente des difficultés en fonction des postes de travail occupés antérieurement.

Dans un troisième temps, nous avons procédé à deux types d'analyses complémentaires:

Une analyse globale qui prend en compte l'ensemble des entretiens. L'objectif étant de repérer des convergences dans les pratiques des écoutants; pour cela, l'ensemble des entretiens a été soumis à un seul traitement.

Une analyse détaillée qui repose sur la distinction entre le discours des écoutants et celui des écoutantes. En raison des difficultés que suppose la relation d'écoute dans ce contexte précis, nous avons cherché à comprendre si les mécanismes mis en œuvre par les écoutants étaient différents selon le genre.

Nous avons complété ces différents résultats avec des extraits d'entretiens notamment ceux qui exprimaient des situations difficilement vécues par les écoutants. Ils ont permis de valider les résultats obtenus.

\section{4.- Résultats}

Les résultats que nous présentons concernent d'une part le discours global des écoutants et d'autre part, une distinction entre les écoutants hommes et femmes. La particularité du discours global c'est de focaliser l'attention sur les difficultés de l'activité et les stratégies des écoutants pour y faire face. L'analyse comparative confirme ce discours en accentuant les points de vue divergents mais complémentaires des écoutants. En effet, les hommes montrent les limites du dispositif d'urgence tandis que les femmes interpellent fortement l'organisation du travail. Les classes de discours se distinguent les unes des autres par le nombre d'uce (unités de contexte lexicaux) que chacune regroupe. Plus ces unités de contexte sont importantes plus la classe occupe une place importante dans la représentation des opérateurs.

\section{1.- Un discours global dominé par l'origine socio-professionnelle des écoutants}

Ce premier niveau de l'analyse nous a permis de repérer cinq classes de discours que nous présentons par ordre d'importance.

La classe 3 reflète le plus l'avis des écoutants. Elle rassemble à elle seule 128 uce, soit (46,5\%) de l'ensemble du discours des écoutants. Il s'agit du discours selon lequel, « Le parcours des écoutants dans le social permet de mieux gérer la relation d'écoute ».

La classe 1 avec 46 uce, soit 16,7 \% du discours global. Il s'agit du discours concernant « La double fonction de l'écoute: expression des personnes sans abris et source de stress pour les écoutants».

La classe 5 «Les écoutants sont particulièrement affectés par la détresse des femmes et des enfants » 
avec 44 uce, représente $16 \%$ du discours global.

La classe 2 «L'obligation pour les écoutants de justifier le manque de place » avec 32 uce, soit $11,6 \%$ du discours.

La classe 4 «Répartition inégale des appels au cours de la journée » avec 25 uce, soit $9 \%$ du discours global des écoutants.

\section{2.- L'analyse comparative}

Ce second niveau d'analyse nous permet d'obtenir deux types de résultats : le discours des écoutants hommes et celui des écoutants femmes.

\subsection{1.- Les hommes face à un dispositif dépassé}

Le sentiment des hommes en charge de l'écoute c'est d'avoir affaire à un dispositif de plus en plus limité. Avec le temps en effet ce dispositif a vu son objectif premier, à savoir proposer un hébergement d'urgence aux appelants, évoluer vers d'autres problématiques sociales. Par ailleurs, la tâche des écoutants est rendue encore plus difficile à partir du moment où les possibilités d'hébergement ne répondent plus aux nombreuses demandes sans cesse renouvelées. Ce qui ne rend pas aisée la relation d'écoute téléphonique. Les quatre classes de discours identifiées résument cette réalité. Si les deux premières relatent les difficultés de leur activité (manque de place, stress), les deux autres évoquent quant à elles les stratégies développées par les écoutants pour réguler cette relation d'écoute.

La classe 2 "Le Non, c'est-à-dire le manque de place, est vécu comme source de souffrance et à l'origine de l'agressivité de certains usagers du 115 », exprime largement le sentiment des écoutants hommes avec 39 uce, soit 39,3\% du discours.

La classe 1 "Le stress des écoutants est la conséquence du manque de place mais aussi l'obligation de justifier ce manque » regroupe 22 uce, soit 22,2\% du discours. Cela s'exprime comme nous l'avons évoqué précédemment, par l'obligation pour les écoutants de justifier ce manque.

La classe 3 «Rôle de l'expérience dans la gestion de l'écoute téléphonique ». Elle regroupe 21 uce, soit $21,2 \%$ du discours.

La classe 4 «La relation d'écoute nécessite une certaine disponibilité », avec 17 uce, soit 17,1\% du discours traduit la capacité des écoutants à faire face à toutes les situations.

\subsection{2.- L'organisation du travail fortement interpellée par les écoutantes}

Le discours des femmes s'articule autour de l'organisation du travail comme principal facteur dans la problématique de la relation d'écoute. Face à la détresse des appelants et la limite des moyens qui leur sont affectés, les écoutantes s'organisent individuellement ou collectivement pour mieux répondre et orienter les sans-abri. Cinq classes d'uce émergent de leur discours.

La classe 1 obtient 37 uce, soit $28,6 \%$ du discours. C'est l'idée selon laquelle «Le personnel féminin est particulièrement affecté par la détresse des femmes et des enfants ». Nous retrouvons ici le même discours que dans l'analyse globale.

La classe 5 recueille à peu près le même nombre d'uce (36), soit 27,9\% du discours. Elle concerne notamment les «Limites du travail des écoutants: nécessité d'un relais sur le terrain par les travailleurs sociaux».

La classe 3 obtient 20 uce, soit 15,5\% du discours. Dans ce discours, ce sont principalement les «Stratégies individuelles et collectives pour réguler le stress du téléphone » qui sont évoquées.

La classe 4 se distingue avec 19 uce, soit 14,7 \% du discours. L'attention est portée sur le «Rôle de l'écoute dans la régulation des phénomènes d'agressivité verbale». 
Enfin, la classe 2 obtient 17 uce, soit 13,1\% du discours. À ce niveau, les écoutants parlent de « L'importance de leur travail dans la problématique des personnes sans abris ».

\section{5.- Quelles significations pour l'activité?}

Cette étude avait pour objectif d'analyser l'activité des écoutants engagés dans une relation d'aide au téléphone, afin d'évaluer leurs difficultés et proposer des solutions sur le plan organisationnel. Pour ce faire, nous avons utilisé la technique de l'entretien semi-directif. Les données obtenues ont été soumises au traitement du logiciel Alceste dont les résultats permettent, pour une large partie, de répondre aux interrogations qui étaient les nôtres tout au long de cette étude.

L'analyse globale montre que les écoutants organisent leur discours majoritairement autour des difficultés liées à leur activité d'une part et sur les stratégies qu'ils mettent en œuvre pour y faire face d'autre part. Au chapitre des difficultés, on note le plus souvent des problèmes institutionnels et organisationnels pour lesquels les écoutants sont démunis : à titre d'exemple, l'objet de travail qui n'est pas clairement défini. Car, contrairement à certains services téléphoniques, le 115 peut être sollicité pour plusieurs raisons: demande d'information, recherche d'hébergement, besoin de soutien moral, etc. Comme cela a pu être indiqué plus haut, les statistiques de ce dispositif montrent que deux appels sur trois ne concernent pas directement l'hébergement. Ce qui oblige les écoutants à s'adapter à chaque situation. Parce que l'objet de la demande n'est pas clairement exprimé au départ, ils vont procéder à la reformulation de la demande afin de pouvoir la ranger dans un modèle qui leur est connu, ceci afin d'apporter une réponse adaptée (Brangier, 2002). Contrairement à ce qui a pu être observé par Engrand (2002) dans la relation d'aide au téléphone sur le cancer, la co-construction de l'activité est rendue parfois plus difficile dans le contexte du 115 dans la mesure où l'objet de la demande n'a pas forcement de rapport direct avec la problématique de l'hébergement. Une pluralité d'objets qui mobilise différemment les écoutants au plan cognitif et les oblige à user de compétences autres que celles requises dans la relation d'écoute. On peut ajouter également les nombreux appels polluants qui paralysent par moments la ligne et empêchent les appels véritables d'aboutir. Un constat qui démontre ainsi la vulnérabilité de ce moyen de communication qui est censé apporter une première réponse aux difficultés des personnes en errance. Enfin, la répartition inégale des appels dans la journée conduit à des pics d'appels pour lesquels les écoutants réagissent parfois dans l'urgence. Ainsi diront-ils: "le stress c'est quand on a plusieurs coups de fil à gérer en même temps. C'est stressant parce qu'il faut gérer l'urgence. Les personnes sont impatientes ».

Les conditions de travail des écoutants ainsi décrites peuvent paraître obsolètes à côté de la saturation du dispositif dans sa capacité à offrir des places adaptées aux personnes sans domicile fixe. À ce propos, la rhétorique selon laquelle « le 115 est victime de son succès» est tout à fait révélatrice de la souffrance des écoutants, en particulier au moment où ils apportent une réponse négative à un appel. " Le Non », c'est-à-dire le manque de place, pose doublement problème: d'une part, il est vécu par eux comme source de souffrance et d'autre part, il est à l'origine de l'agressivité de certains usagers insatisfaits. En effet, les écoutants disent ne pas savoir à quelle réaction ils peuvent être exposés lorsqu'ils déclarent par moments ne plus avoir de place. La souffrance est ainsi liée à la qualité de la réponse apportée à l'appelant: " c'est assez dur de dire Non. Je comprends la réaction de personnes qui le prennent mal, qui réagissent de façon agressive et qui ne comprennent pas qu'on leur dise d'appeler mais à chaque fois, on leur dit qu'il n'y a pas de place. C'est frustrant pour eux, mais aussi pour nous ». La souffrance engendrée par l'incertitude du comportement de l'usager est d'autant plus grande que les écoutants doivent justifier le manque de place sur le dispositif à certains moments de la journée, sous peine d'entrer en conflit avec l'appelant. Certains usent parfois de pédagogie pour faire comprendre que, contrairement à ce qui est véhiculé par les médias, les moyens du 115 sont limités: " nous prenons sur nous la responsabilité d'expliquer ce manque de place. J'essaie de transformer l'image toute puissante qu'ils ont du 115 pour leur montrer qu'on a aussi des limites, on ne peut pas tout faire $\gg$. 
Parmi les facteurs de régulation de leur activité, les écoutants se sont prononcés largement sur le parcours professionnel et sur la méthode de l'écoute elle-même, leur principal outil de travail. Beaucoup d'entre eux ont déjà exercé dans le milieu associatif en utilisant le plus souvent la technique de l'entretien comme outil d'évaluation. Ainsi, ils mettent à contribution des compétences acquises ailleurs, par exemple dans des structures d'écoute comme Sida info service, ou dans des associations spécialisées dans la réinsertion sociale d'adolescents ou d'adultes. D'autres par contre poursuivent encore des études de la filière psychologie et profitent du dispositif pour acquérir des connaissances supplémentaires. En somme, une culture sociale variée qui aurait permis à l'ensemble des écoutants de mieux gérer la relation d'écoute, ce d'autant plus que ce n'est que récemment ${ }^{4}$, qu'un référentiel métier de l'écoute sociale a été élaboré. Une autre carence dans ce domaine c'est aussi l'inexistence de formation continue de l'écoute sociale. Car, en raison de son coût élevé, la formation dans le domaine de l'écoute est proposée aux Associations sous la forme de sessions, par les organismes agréés $^{5}$. Le rôle de l'écoute téléphonique dans la régulation de cette activité tient à la fonction sociale qu'elle remplit pour les personnes sans abri, bien qu'elle constitue un coût pour les écoutants. Elle permet de maintenir le fragile lien qui existe encore entre les personnes sans abris et la société. L'écoute téléphonique est perçue comme moyen d'expression des personnes sans abri. Ce qui conduit les écoutants à développer de l'empathie. Comme ils le déclarent, "l'objet téléphonique apparaît comme le seul lien qui permet à ces personnes d'être encore avec la société. Beaucoup, sont vraiment perdus, désocialisés. Chaque coup de fil est une rencontre avec quelqu'un qui exprime quelque chose, un besoin, ou quelque chose de sa souffrance, de sa colère, de sa peine, ou de sa déprime ». Mais parfois, l'écoute est à l'origine du stress des écoutants. Certains appels sont ressentis en effet comme des appels au secours pour lesquels les écoutants manquent de formation et de soutien lorsque se présentent des situations difficiles. Ce qui leur donne l'impression d'être parfois persécutés par les usagers: " il arrive qu'une personne appelle et dit qu'elle va se suicider. Moi, personnellement ça me déstabilise. Je ne sais pas quoi dire. C'est de la violence au sens où je me demande pourquoi c'est à moi que ça arrive?".

Si l'analyse globale du corpus renseigne sur les difficultés de l'activité des écoutants et les stratégies qui y sont déployées, l'analyse comparative permet de prendre la mesure de cette réalité selon que l'écoute se fait par un homme ou une femme. Ainsi, le sentiment des hommes c'est d'être face à un dispositif dépassé. Leur discours s'étend largement sur les limites du dispositif d'urgence sociale et sonne comme une révolte. En raison principalement des nombreuses demandes qui ne peuvent être quotidiennement satisfaites, ce dispositif ne semble plus remplir correctement le rôle pour lequel il a été créé. Dans ces conditions, les écoutants sont pris dans des logiques contradictoires qui les obligent à avoir non seulement une certaine disponibilité vis-à-vis des appelants mais aussi d'épuiser toutes leurs ressources: "le travail au 115 demande de prendre beaucoup sur soi; nous avons exigence de satisfaire au maximum aux demandes qui nous sont adressées; nous devons beaucoup de disponibilité aux personnes sans abri. Est-ce au-delà de nos missions? Ca fait partie intégrante du lien social. C'est une façon de positiver son travail, surtout lorsque l'on y travaille depuis de nombreuses années ». Face à ces situations, les hommes indiquent le rôle de l'expérience et de leur disponibilité comme stratégies dans la gestion de l'écoute téléphonique. Par exemple, ils déclarent comprendre tout à fait le comportement décalé de certains usagers: "je comprends la réaction des gens qui le prennent mal et qui réagissent de façon agressive quand on leur dit qu'il n'y pas de place». À terme, ces moments difficiles finissent par être acceptés par les écoutants et reconnus comme partie intégrante de leur activité: «J'ai l'impression de me blinder; je ne me laisse pas trop aller à mes sentiments. Je me contente de rester dans mon rôle d'intervenant et je dis simplement qu'on n'a pas de place ». Un sentiment d'impuissance et une stratégie d'action qui n'est pas sans rappeler le type de comportement décrit par De Gaulmyn $(1994 ; 2002)$ au sujet des écoutants qui cachent leurs

4. Le seul référentiel connu de nos jours est contenu dans un programme plus vaste élaboré en mars 2005 par la DDASS et qui concerne «l'Accueil, l'Hébergement et l'Insertion ». Pour certaines Associations, il ne reflète pas la réalité sur le terrain.

5. L'Ecole des Parents et des Educateurs (EPE) propose à Paris ce genre de formation aux Associations spécialisées dans l'accompagnement social des personnes en difficulté. 
émotions vis-à-vis des usagers, pour éviter de se faire humilier.

La position adoptée par les écoutants hommes est plus nuancée chez les écoutantes, tant sur le mode opératoire que sur la perception de leurs conditions de travail. Pour ce qui les concerne, l'organisation du travail apparaît à leur avis comme principale cause de leurs difficultés. Conscientes des réels problèmes que rencontrent les pouvoirs publics à satisfaire l'ensemble des demandes en matière d'hébergement, les écoutantes considèrent que le peu de places disponible doit être savamment géré en fonction des problématiques. C'est le cas notamment de la détresse des femmes et des enfants dont elles déclarent être particulièrement affectées. En effet, beaucoup d'appels concernent des cas où des femmes seules ou accompagnées d'enfants sont victimes de violence conjugale et livrées à elles-mêmes. Plus qu'un simple processus d'identification, la sensibilité des écoutantes vis-à-vis des problèmes de cette catégorie d'usagers doit être interprétée par le fait que les usagers ne sont pas tous égaux dans la rue où les femmes et les enfants qui les accompagnent sont sans aide et s'exposent à de nombreux dangers. De telles situations sont d'autant plus préoccupantes que les prises en charge (solutions d'hébergement) proposées ne sont pas adaptées le plus souvent à ce genre de problématique sur le dispositif. Beaucoup de ces femmes sortent fraîchement des hôpitaux et se retrouvent hébergées en collectif, sans mesures d'accompagnement adaptées à leur situation de convalescence. Les écoutantes reconnaissent également l'existence des phénomènes d'agressivité verbale dans la relation avec les usagers même si, à leur avis, l'écoute téléphonique joue un rôle considérable dans la régulation de cette agressivité: «Des fois, il y a des pleurs dans les appels. C'est quelque chose d'assez difficile à gérer. Je laisse le temps à la personne d'évacuer. Quand ce sont des insultes, je considère qu'elles ne me sont pas adressées. La personne finit par raccrocher ». Comme cela transparait, le travail des écoutants exige une part importante maîtrise de soi. Parce qu'il se sait écouté, l'usager finit par changer de comportement envers l'écoutant. La garantie pour un tel résultat tient beaucoup donc de la position qu'adoptent les écoutantes. Néanmoins, en raison des affects qu'ils contiennent (pour lesquels les écoutants sont souvent démunis, faute de formation adaptée), les appels peuvent conduire à la longue à de la lassitude: "ça peut être des appels pour lesquels même après la conversation, je continue de penser ou bien quej'ai besoin d'une pause pour pouvoir décompresser. Il y a de la fatigue psychique et morale autour de ça. Il arrive même que j'aie besoin d'en parler ». Enfin, les écoutantes évoquent constamment l'importance de leur travail dans la problématique des personnes sans abris. Toutefois, comme elles le reconnaissent, cette aide ponctuelle a ses limites. L'écoute et le soutien au téléphone n'auront de réels effets que s'ils sont relayés sur le terrain par d'autres spécialistes du social. C'est la raison pour laquelle les écoutantes expriment la nécessité que les personnes sans abri soient accompagnées au cours de leurs démarches d'insertion par les travailleurs sociaux: " avoir un réfèrent social c'est quelque chose d'essentiel pour les usagers, parce que c'est lui qui va faire le pont entre les différents interlocuteurs ». Une solution qui aboutit pourtant très rarement à cause de la grande mobilité des travailleurs sociaux et de leur nombre insuffisant.

Bien que portant sur les conditions de travail, le discours des écoutants renvoie dans son ensemble aux nombreux travaux sur l'implication affective (Bange, \& De Gaulmyn, 1990; De Gaulmyn; 1994 ; 2002) selon lesquels tout professionnel du social est impliqué dans des transactions interpersonnelles, en affrontant des situations plus ou moins éprouvantes et susceptibles d'ébranler les composantes/dimensions émotionnelles de son équilibre psychique (Villate, Logeay, Mabit, \& Pichenot, 1989; Villate et al., 2004). Ce cas particulier des écoutants peut être rapproché de celui plus général des travailleurs sociaux (Pezet-Langevin, 1997, 2001; Trichot, 2004), mais aussi du personnel soignant en contact avec des malades (Landau, 1992; Saint-Arnaud, Gingras, Boulard, Vésina, \& LeGosselin, 1992), situations dans lesquelles les auteurs ont mis en évidence le problème de la distance observée pendant l'écoute, pour réguler la relation entre le professionnel et son interlocuteur. Ainsi, certains auteurs pensent que cette distance doit être relative (Villate et al., 2004). Autrement dit, elle ne doit pas être ni trop grande (pour éviter la froideur pouvant être interprétée comme manque d'intérêt), ni trop courte (risque d'implication affective dans la relation qui pourrait perdre son caractère professionnel). Pour pouvoir mettre en pratique une telle mesure, il est recommandé de pouvoir bénéficier de soutien. Par exemple, comme l'indique Pezet-Langevin (2001), les éducateurs spécialisés 
dans l'accompagnement de jeunes en difficultés sont aidés par des psychologues vacataires dans leur travail au quotidien. Mais selon les besoins et en accord avec l'association où ils évoluent, les éducateurs peuvent bénéficier d'un soutien personnel également.

En conclusion, l'analyse du discours par le logiciel Alceste confirme la possibilité d'étudier l'activité d'opérateurs intervenant dans le secteur social par les outils réajustés que sont les entretiens (Vermersch, \& Maurel, 1997; Valléry, 2000; Wolff, \& Visser, 2004; Djibo, Valléry, \& Lancry, 2006). Ainsi, si dans la globalité l'outil d'analyse permet de comprendre les représentations que les écoutants se font de leur activité, l'analyse détaillée du corpus permet de confronter le point de vue des écoutants hommes et femmes et de mettre en évidence par la même occasion l'existence d'une différence de mode opératoire entre eux. Comme on le perçoit, les différences de modes opératoires sont fonction de la sensibilité propre aux écoutants mais aussi de leur genre, confirmant ainsi le point de vue développé par Messing (1999), c'est-à-dire la pertinence de tenir compte du sexe des opérateurs dans les études ergonomiques.

Du point de vue organisationnel, cette étude met en évidence les difficultés d'une situation de travail dont les caractéristiques dépassent les seules ressources des écoutants. En effet, la problématique du logement d'urgence est un enjeu national. Cependant, sa gestion au plan local repose sur un réseau partenarial entre les centres d'hébergement et le service téléphonique qui gère les appels des usagers. Si une telle organisation peut paraitre incontournable au vu de l'importance du problème du logement d'urgence, elle aura toutefois démontré ses limites de nos jours. Car, l'activité des écoutants repose sur un certain nombre de contradictions en relation directe avec la pratique de l'écoute. Cette activité dépend énormément de variables telles que le flux des appels inégaux en journée, la nature des demandes différente à chaque fois, mais aussi le contenu de certains appels, soit chargés d'émotions, soit agressifs. On peut aisément comparer cette problématique à celle des centres d'appels où, selon Grosjean et Ribert-Van de Weerdt (2005), la relation avec le client, bien que paraissant au premier plan chargée d'émotions, montre en réalité que les salariés souffrent davantage des modalités de management et de contrôle. Notre analyse a montré qu'en pareilles situations il existe des variations individuelles et collectives quant aux stratégies déployées par les écoutants pour faire face. Cela met en évidence la nécessaire formation des écoutants mais aussi l'harmonisation des pratiques dans ce domaine, dans la mesure où ceux-ci doivent faire appel à des compétences variées (relationnelles, linguistiques) et gérer par la même occasion des conflits intrapsychiques. L'étude montre en effet des problématiques de gestion de la relation d'écoute dont on sait qu'elle dépasse le simple registre du bénévolat. Elle fait également ressortir le souhait exprimé par les écoutants de disposer d'un espace de parole où exprimer les ressentis sur des situations vécues plus ou moins difficilement et le besoin de renforcer la dimension collective du travail, afin que les écoutants puissent s'épauler en cas de besoin. Il ressort enfin qu'un travail important doit être fait dans le sens du renforcement des équipes pour pouvoir réguler les flux des nombreux appels qui, s'ils n'aboutissent pas, peuvent être à l'origine de l'agacement de certains usagers.

\section{RÉFÉRENCEMENT}

Djibo, S. (2008). Contribution d'une analyse du discours à l'étude des stratégies d'actions et de régulation des agents en situation d'écoute téléphonique: 1'exemple du dispositif d'urgence sociale 115. @ctivités, 5 (1) pp. 3-20, http://www.activites.org/v5n1/v5n1.pdf

\section{BIBLIOGRAPHIE}

Bange, P., \& De Gaulmyn, M. (1990). Études conversationnelles d'appels téléphoniques d'urgence. Mission Interministérielle de Recherche et d'Expérimentation (MIRE), Ministère des Affaires Sociales.

Boucheix, J.M. (1991). L'accueil au commissariat de police. Journées d'études, Centre national d'étude et de formation (CNEF) de la police nationale, Gif-sur-Yvette. 
Brangier, E. (2002). Le travail conversationnel: analyse de l'activité conversationnelle dans le « call center» d'une compagnie d'assurance. In E. Engrand, S. Lambolez, \& A. Trognon (Eds.), Communications en situation de travail à distance (pp. 28-48). Nancy: PUN.

Cadéac, B., Ferrarini, Y., \& Oddoux, K. (2004). L'éducation pour la santé peut aussi passer par le téléphone. La santé de l'homme, INPES, $\mathrm{N}^{\circ} 373,7$.

Caillat, S. (2005). Le drame de l'hôtel Paris-Opéra ravive le débat sur l'hébergement d'urgence. 20 minutes, quotidien d'information gratuit, lundi 18 avril 2005.

Cerf, M., \& Falzon, P. (2005). Situations de service: travailler dans l'interaction. Paris: PUF.

Chambras, A. (2004). Le 115 ne répond plus. Actualités Sociales Hebdomadaires (ASH), N 2378 du 22-10-2004.

Chauvière, M. (2001). Ecoutant, un champ professionnel en gestation, convergences téléphoniques. Revue de la téléphonie sociale et économique, 1, 40-51 (Les métiers de la téléphonie), diff. L'Harmattan.

Chauvière, M. (2002). La téléphonie sociale entre bénévolat et salariat: réflexion sur un paradoxe. Revue française des affaires sociales, 63-71.

Chopart, J.-N. (Ed.) (2000). Les mutations du social. Dynamiques d'un champ professionnel. Paris: Dunod.

Davidson, J.-C., Francescon, J.-M., Negroni, P., Pezet-Langevin, V., \& Rolland, J.-P. (1995). L'usure professionnelle des travailleurs sociaux: une triple approche: psychologique, ergonomique et psychodynamique. Paris: Editions INPACT.

De Gaulmyn, M.M. (1994). Appels téléphoniques d'urgence sociale: parler au nom d'un autre. In A. Trognon, \& C. Riboni (Eds.). Construction interactive du quotidien (pp. 235-261). Nancy: PUN.

De Gaulmyn, M.M. (2002). L'émotion au téléphone. De la perception à la reformulation. In E. Engrand, S. Lambolez, \& A. Trognon (Eds.), Communications en situation de travail à distance (pp. 84-99). Nancy: PUN.

Dejours, C. (1993). Travail et usure mentale. Paris: Bayard.

Delignères, G. (2002). La construction sociale d'une profession ou d'un métier à travers son évaluation identitaire: essai sur l'activité de téléopérateur en France. Mémoire DEA, Université Pierre Mendès France, Grenoble 2.

Demoulin, J (1996). L'écoute téléphonique urgente, Le temps à l'adolescence. Journées scientifiques du 12 octobre 1996. In N. Pierre, \& N. Poitou (Eds.), La santé de l’homme, INPES (2004), n³73, 13-15.

Djibo, S., Valléry, G., \& Lancry, A. (2006). Charge mentale et régulation de systèmes complexes : approche subjective des agents de régulation du métro parisien. @ctivités, 3 (1), 117-138. http://www.activites.org/

Engrand, E. (2002). Gestion conversationnelle d'un diagnostic médical dans un service d'écoute sur le cancer. In E. Engrand, S. Lambolez, \& A. Trognon (Eds.), Communications en situation de travail à distance (pp. 66-81). Nancy: PUN.

Falzon, P., \& Lapeyrière, S. (1998). L'usager et l'opérateur: ergonomie et relation de service. Le Travail Humain, 61 (1), 69-90.

Falzon, P. (1994). Dialogues fonctionnels et activité collective. Le Travail Humain, 57, 299-312.

Favard-Drialud, A.-M. (1991). L'évaluation clinique en action sociale. Paris: Eres.

FNARS (2003). La santé mentale, hébergement et logement. Actes de la journée du 26 mai 2003.

FNARS (2004). Rapport d'activité, 2003-2004.

Gbézo B. (2000). Agressivité et violence au travail. Paris: ESF Editeur.

Grosjean, V., \& Ribert-Van de Weerdt, C. (2005). Vers une psychologie ergonomique du bien-être et des émotions : les effets du contrôle dans les centres d'appels. Le Travail Humain, 68 (4), 355-378. 
Lambolez (2002). Analyse interlocutoire d'une situation d'assistance informatique par le telephone. In E. Engrand, S. Lambolez, \& A. Trognon (Eds.), Communications en situation de travail à distance (pp. 115-129). Nancy: PUN.

Lancry-Hoestland, A. (2002). Pistes pour l'analyse du travail d'emplois de services d'aide aux personnes.In E. Engrand, S. Lambolez, \& A. Trognon (Eds.), Communications en situation de travail à distance (pp. 131-156). Nancy: PUN.

Landau, K. (1992). Psycho-physical strain and the burn-out phenomen amongst health acre professionnals. In M. Estryn-Behar, Ch. Gadbois, \& M. Pottier (Eds.), Ergonomie à l'hôpital (pp. 331-337). Toulouse: Octarès Edition.

Lechat, N., \& Delaunay, J-C. (2003). Les centres d'appels : un secteur en clair-obscur. Paris: L'Harmattan, recherches économiques.

Lenhnisch, E. (1986). L'aide au téléphone, une nouvelle réponse sociale. Paris: ESF Edition.

Marchand, P., \& Navarro, C. (1994). Analyse de l'échange verbal en situation dialogue fonctionnel: étude de cas. Le Travail Humain, 57, 235-258.

Messing, K. (1999). La pertinence de tenir compte du sexe des «opérateurs» dans les études ergonomiques: Bilan de recherches. Pistes, 1 (1). http://www.pistes.uqam.ca/v1n1/articles/v1n1a5.htm

Minondo, B. (2002). Le contrôle de la compréhension du permanencier du S.A.MU en situation d'interaction téléphonique. In E. Engrand, S. Lambolez, \& A. Trognon (Eds.), Communications en situation de travail à distance (pp. 195-218). Nancy: PUN.

Montheil (1997). Ecouter l'angoisse. Paris: l'esprit du temps.

Pezet-Langevin, V. (1997). Le stress au travail: des déclarations à l'observation des comportements (le syndrome de burn-out chez les travailleurs sociaux). Université Paris X-Nanterre, Thèse de doctorat en psychologie.

Pezet-Langevin, V. (2001). Le burn-out comme manifestation aiguë du stress professionnel. In M. Neboit, \& N. Vézina (Eds.), Santé au travail: le stress professionnel (chapitre 6). Paris: PUF.

Pliez, E. (2003). Errance et santé mentale. In Santé mentale, hébergement et logement. Actes de la journée du 26 mai 2003, FNARS, Ile-de-France, Paris.

Reinert, M. (1987). Un logiciel d'analyse lexicale (ALCESTE). Cahiers d'Analyse des Données, 4, 471-484.

Saint-Arnaud, L., Gingras, S., Boulard, R., Vésina, M., \& Le-Gosselin (1992). Les symptômes psychologiques en milieu hospitalier. In M. Estryn-Behar, Ch. Gadbois, \& M. Pottier (Eds.). Ergonomie à l’hôpital (pp. 338-342). Toulouse: Octarès Editions.

Trichot, (2004). Epuisement professionnel et burn-out: concepts modèles et interventions. Paris: Dunod.

Trognon, A., \& Kostulski, K. (2000). La logique interlocutoire et l'analyse des situations de travail collectif. In T.H. Benchekroun, \& A. Weil-Fassina (Eds), Le travail collectif: perspectives actuelles en ergonomie (pp. 71-96). Toulouse: Octarès Editions.

Trognon, A. (2002). Dialogues et polylogues dans le travail à distance. In E. Engrand, S. Lambolez, A. Trognon (Eds.), Communications en situation de travail à distance (pp. 9-13). Nancy: PUN.

Valléry, G. (2000). Les relations de service dans les organismes publics : stratégies d'action et de régulations des agents face à l'usager. In B. Gandoff (Ed.), L'individu et les performances organisationnelles (pp. 137-151). Paris: L'Harmattan.

Vermersch, P., \& Maurel, M. (1997). Pratiques de l'entretien d'explication. Paris: ESF.

Villate, R., Logeay, P., Mabit, A., \& Pichenot, J.-C. (1989). Les soignants et la mort. Etude de psychopathologie du travail et implications psychopédagogiques. Paris: Institut pour l'amélioration des conditions de travail. Rapport d'étude pour la MIRE, Ministère des Affaires sociales et de l'Emploi.

Villatte, R., Teiger, C., \& Caroly-Flageul, S. (2004). Le travail de médiation et d'intervention sociale. In P. Falzon (Ed.). Ergonomie (pp. 584-601). Paris: P.U.F. 
Wolff, M., \& Visser, W. (2004). Méthodes et outils pour l'analyse des verbalisations : une contribution à l'analyse du modèle de l'interlocuteur dans la prescription d'itinéraires. @ctivités, 2 (1), 99-122. http:// www.activites.org/

\section{RÉSUMÉ}

Dans cette étude, nous avons analysé les représentations d'opérateurs de la téléphonie sociale en particulier les écoutants du numéro d'urgence 115 , afin d'évaluer les difficultés liées à leur activité de travail et les stratégies qu'ils mettent en œuvre pour y faire face. Cette analyse a été faite sur la base du recueil d'entretiens portant sur le vécu des écoutants. La relation d'aide au téléphone met en œuvre de multiples compétences acquises dans des domaines variés. Toutefois, elle apparaît fondée sur de multiples paradoxes qui impactent considérablement l'activité des écoutants. D'autres facteurs d'ordre organisationnel contribuent également à rendre difficile ce travail: de nombreux appels polluants sans relation directe avec leur activité, la dépendance du dispositif à d'autres structures du secteur social, etc. Dans ce contexte, les écoutants développent des stratégies individuelles et collectives pour mieux gérer les contraintes de certains appels ou bien le déficit en places du dispositif d'hébergement.

\section{MOTS CLEFS}

téléphonie sociale; analyse du discours; personnes sans abri; stratégies d'adaptation; écoutants 115.

\section{RESUMEN}

Contribución de un análisis del discurso al estudio de las estrategias de acción y de regulación de los agentes en situación de escucha telefónica: el ejemplo del dispositivo de urgencia social 115. En este estudio hemos analizado las representaciones de los teleoperadores del número de urgencia 115 del servicio de telefonía social, a fin de evaluar las dificultades relacionadas a su actividad de trabajo y las estrategias que emplean para afrontarlas. Este análisis fue realizado a partir de entrevistas destinadas a relevar lo vivido por los teleoperadores. La relación de ayuda telefónica implica el despliegue de múltiples competencias adquiridas en diversos ámbitos. Sin embargo, ésta aparece fundada en diversas paradojas que impactan considerablemente la actividad de los teleoperadores. Asimismo, otros factores de carácter organizacional, contribuyen a dificultar este trabajo: numerosos llamados contaminantes sin relación directa con su actividad, la dependencia del dispositivo con respecto a otras estructuras del sector social, etc. En este contexto, los teleoperadores desarrollan estrategias individuales y colectivas para gestionar más adecuadamente las exigencias que plantean ciertos llamados o el déficit de plazas que ofrece el dispositivo de albergue de las personas sin techo.

Palabras Clave

Telefonía social; análisis del discurso; personas sin techo; estrategias de adaptación; teleoperadores 115

Article soumis le 6 avril 2006 accepté pour publication le 29 octobre 2007 